\title{
Manejo complicaciones biliares de la cirugía del quiste hidatídico hepático
}

\author{
Pedro Pablo Pinto G. ${ }^{1}$ y Jorge Finlez ${ }^{2}$
}

'Servicio de Cirugía Hospital Regional de Coyhaique. Coyhaique, Chile.

2Departamento de Producción Hospital Regional Coyhaique. Coyhaique, Chile.

Recibido el 12 de diciembre de 2019 y aceptado para publicación el20 de enero de 2020

Correspondencia a: Dr. Pedro Pablo Pinto G. pedropablopatricio@gmail.

\section{Management of biliary complications of liver hydatid cyst surgery}

The most frequent complication of hepatic hydatid cyst is the external biliary fistula. Pre and intraoperative diagnosis is essential in treatment. This evaluation describes predictive factors that make cystobiliary communications suspicious. In the presence of these it is possible to carry out additional studies for its diagnosis. Filtration and biliary fistula are the most frequent postoperative complications and the main causes of morbidity and mortality. Their rates can be decreased by acutely following the predictive factors and proper management of postoperative biliary fistula.

Key words: echinococcosis; hepatic hydatid cyst; hydatid surgery; biliary filtration; biliary fistula.

\section{Resumen}

La complicación más frecuente del quiste hidatídico hepático es la fístula biliar externa. El diagnóstico pre e intraoperatorio es esencial en el tratamiento. En dicha evaluación se describen factores predictivos que hacen sospechar las comunicaciones cistobiliares. En presencia de estas, es posible realizar estudios adicionales para su diagnóstico. La filtración y la fístula biliar son las complicaciones postoperatorias más frecuentes y las principales causas de morbimortalidad. Sus tasas pueden disminuirse siguiendo con acuciosidad los factores predictivos y el adecuado manejo de la fístula biliar postoperatoria.

Palabras clave: echinococcosis; quiste hidatídico hepático; cirugía hidatídica; filtración biliar; fístula biliar.

\section{Introducción}

El Echinococcus granulosus afecta al hígado en aproximadamente el $70 \%$ de los casos, y sigue siendo una de las principales indicaciones de cirugía hepática en áreas endémicas ${ }^{1}$. La información existente sobre tasas de mortalidad relacionadas con la hidatidosis se encuentra entre un 0,5 y $4 \%$, la mayor parte a consecuencia de las complicaciones ${ }^{1,2}$.

La cirugía ya sea de la manera tradicional o laparoscópica, tiene dos modalidades: radical o conservadora. La forma radical tiene como objetivo la extirpación completa del quiste y la periquística; la periquistectomía y la hepatectomía son ejemplos (Figura 1). Estas tienen tasas más bajas de complicaciones $(0-26 \%)$ y recurrencia $(0-3 \%)$ que los métodos conservadores ${ }^{1,3}$. Para este tipo de cirugía se requiere de experiencia quirúrgica hepatobiliar y de un tiempo de operación más prolongado, lo que constituye una severa limitación en regiones endémicas. Por el contrario, los enfoques conservadores
(Figura 2), tales como la periquistectomía parcial con drenaje u omentoplastía, requiere de menos experiencia y de tiempos quirúrgicos menores. Sin embargo, sus tasas de complicaciones (6-47\%) y recurrencia (4-25\%) son más altas ${ }^{3}$.

La filtración biliar y la formación de fístulas postoperatorias, son resultado de la comunicación cistobiliar (CCB) debido a la ruptura intrabiliar. La incidencia de filtración de bilis postoperatoria se encuentra entre 2,5 y $28,6 \%$. Cuando la filtración de bilis postoperatoria se drena externamente y persiste por más de 10 días, se denomina fístula biliar. Es la mayor causa de hospitalización prolongada y de complicación de esta cirugía ${ }^{3,4}$.

Las fístulas en su gran mayoría cierran espontáneamente en la primera semana del postoperatorio. Estas se pueden clasificar como de alto y bajo flujo dependiendo de la cantidad de drenaje biliar. Se dice que es de alto flujo cuando el drenaje diario es $>$ a $300 \mathrm{ml} /$ día y de bajo flujo cuando es menor a dicha cantidad $^{4,5}$. 

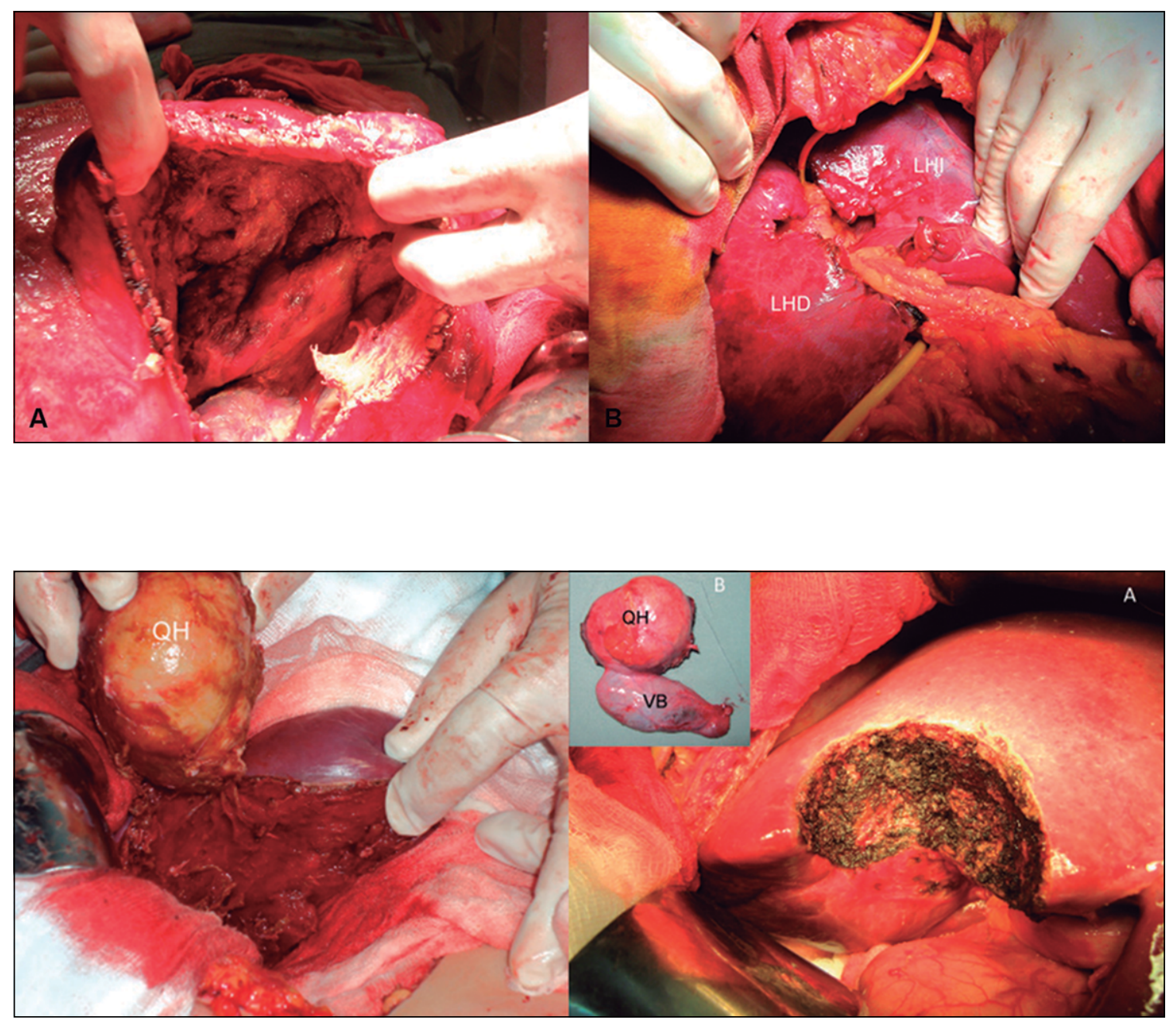

Se han propuesto dos teorías para la patogenia ${ }^{6}$ (Figura 3).

- La primera, es la necrosis progresiva que se desarrolla en la pared del conducto biliar debido a la compresión ocasionada por el quiste hidatídico.

- La segunda, sugiere que los conductos biliares pequeños, que están atrapados dentro de la membrana periquística, se atrofian debido a la presión quística lo que con lleva finalmente a su rotura.

A pesar que algunos estudios establecen tasas de comunicación de hasta $90 \%$ durante la evolución de los quistes, la incidencia de CCB clínica es sólo de $13-37 \% 0^{4,6}$.

Hay dos tipos de CCB: la franca $(>5 \mathrm{~mm})$ y la oculta $(<\text { de } 5 \mathrm{~mm})^{6}$.

- La CCB franca, es una comunicación amplia entre el quiste y el sistema biliar. Como la presión intraquística es mayor que la presión biliar, la CCB franca, permite que los contenidos quísticos, como los quistes hijos o los fragmentos de membrana, ingresen en el sistema biliar y produzcan síntomas clínicos, tales como ictericia obstructiva, colangitis, infección del quiste o anafilaxis. Su incidencia varía entre un 3 y $17 \% 0^{3,6}$. La CCB franca se puede diagnosticar antes de la operación $\mathrm{y}$, en consecuencia, se podrá planificar su tratamiento.

- La CCB oculta, es una comunicación más reducida entre el quiste y los radicales biliares. $\mathrm{Su}$ incidencia varía entre el 10 y el $37 \%$ de los pacientes con quiste hidatídico hepático ${ }^{3,7}$. Por lo general, es asintomática en el período preoperatorio y, por lo tanto, difícil de diagnosticar.
Figura 1. Cirugía conservadora. A: quistectomía parcial. B: quistectomía + omentoplastía. LHD: lóbulo hepático derecho. LHI lóbulo hepático izquierdo.

Figura 2. Quistectomía total cerrada. QH: quiste hidatídico. VB: vesícula biliar 


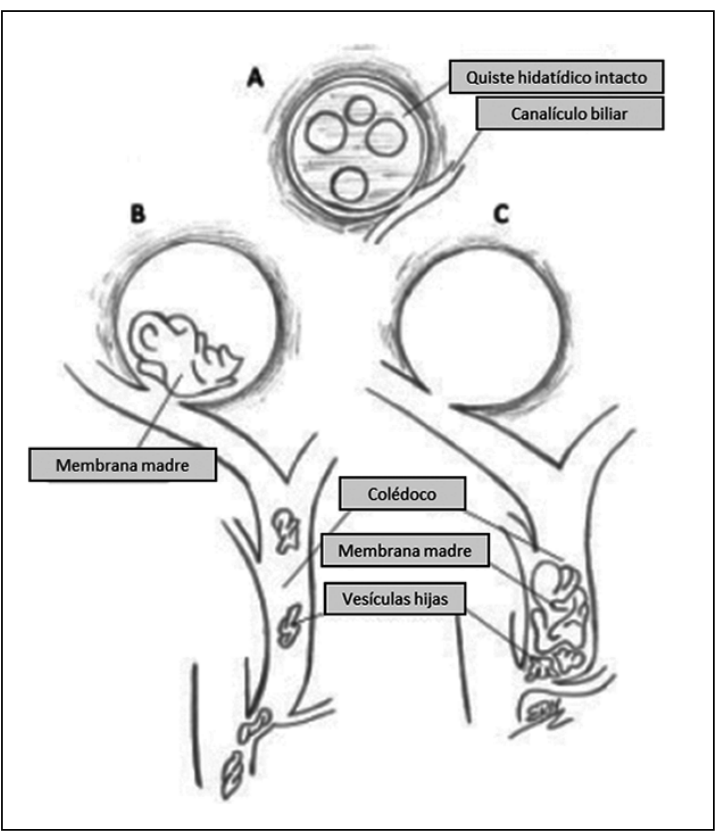

Figura 3. Quiste hidátidico hepático abierto en vías biliares. A) Comunicación con pequeño canalículo biliar. B) Evacuación parcial con pasaje de vesículas hijas. C) Evacuación total.

\section{Evaluación preoperatoria}

\section{- Evaluación de CCB franca}

Puede ser diagnosticada preoperatoriamente.

En un quiste no roto, la presión intraquística está entre 30 y $80 \mathrm{~cm}$ de $\mathrm{H}_{2} \mathrm{O}$, mientras que la presión intrabiliar normal es de 15 y $20 \mathrm{~cm} \mathrm{H}_{2} \mathrm{O}$. Cuando se desarrolla una CCB franca, los materiales intraquísticos, tales como los quistes hijos y los fragmentos de membrana, entran en los conductos biliares como consecuencia de la gradiente de presión hacia el sistema biliar. El material hidatídico provoca un cuadro de ictericia obstructiva, que se observa en un 57 a $100 \%$ y la colangitis en un 20 a $37 \%$ de los pacientes. Sin tratamiento, ambos pueden provocar sepsis y muerte, con tasas de mortalidad tan altas como el $50 \%{ }^{7}$.

En 30 al $74 \%$ de los casos, el diagnóstico se puede realizar mediante ecografía ${ }^{6}$. Las estructuras ecogénicas lineales irregulares, sin sombra acústica en el conducto biliar y un sistema biliar dilatado, son hallazgos ecográficos en casos de CCB franca ${ }^{3}$.

La sensibilidad de la tomografía axial computada es de $75 \%{ }^{6}$. A través de ella es posible visualizar materiales hidatídicos en los conductos biliares o un defecto de la pared quística.

La colangiopancreatografía por resonancia mag-
Tabla 1 Predictores clínicos CCB franca

Antecedente de ictericia
Localización central de quistes $>10 \mathrm{~cm}$
Presencia de hallazgos sugerentes a la ultrasonografía
Quistes tipo CE II y IV (clasificación OMS-IWGE)

nética (CPRM) es otro método alternativo y más eficaz para el diagnóstico preoperatorio en casos sospechosos. Este examen tiene una sensibilidad de $92 \%$ y una especificidad de $83 \%{ }^{6}$.

\section{Predictores clínicos de CCB franca ${ }^{7}$ (Tabla 1)}

- Antecedente de ictericia.

- Localización central de quistes $>10 \mathrm{~cm}^{8}$.

- Presencia de hallazgos sugerentes a la ultrasonografía.

- Quistes tipo CE IV (clasificación OMS-WGE).

En casos de sospecha clínica y presencia de factores predictivos, se puede planear estudios adicionales como la CPRM o la colangiopancreatografía retrógrada (CPRE). Debido a que la CPRM es una prueba no invasiva, es posible utilizarla como método primario para el diagnóstico. Después de la confirmación del diagnóstico de $\mathrm{CCB}$, se puede utilizar la CPRE para el tratamiento.

La CPRE, se puede utilizar tanto para fines diagnósticos como terapéuticos ${ }^{9,10}$. Tiene una sensibilidad diagnóstica de 86 a 100\% para CCB franca. Sirve para:

- Tratar afecciones agudas y diferir, por ende, la cirugía.

- Disminuir las tasas de complicaciones postoperatorias y lograr un tiempo de hospitalización postoperatorio más corto.

- Evacuación de contenidos hidatídicos biliares y quísticos.

La realización de CPRE con esfinterotomía endoscópica previa a la cirugía, disminuye el riesgo de fístula biliar postoperatoria de 11,1 a un 7,6\%. Además, el 25\% de los pacientes con CCB franca, se mejoraron solo con la realización de la CPRE, sin requerir cirugía adicional.

\section{- Evaluación de CCB oculta}

La CCB oculta, es difícil de diagnosticar antes de la operación ya que, generalmente, no es posible visualizar una pequeña comunicación en los estudios radiológicos ${ }^{7,11,12}$. La mayoría de los casos 
Tabla 2. Predictores clínicos CCB oculta

\section{Nivel de ALP > $144 \mathrm{U} / \mathrm{L}$}

Nivel de gamma-glutamiltransferasa (GGT) $>34,5 \mathrm{U} / \mathrm{L}$

Nivel total de bilirrubina $>0,8 \mathrm{mg} / \mathrm{dL}$

Nivel total de bilirrubina directa $>0,4 \mathrm{mg} / \mathrm{dL}$

Diámetro de quiste $>8,5 \mathrm{~cm}$

Quistes tipo CE II y IV (WHO-IWGE)

son asintomáticos hasta la cirugía o en el período postoperatorio cuando se presenta la fístula biliar.

Durante una operación bajo la modalidad conservadora, la tinción biliar del contenido quístico no se visualiza en la mayoría de los casos. Una vez que la gradiente se invierte con la aspiración y evacuación del contenido quístico, comienza la filtración biliar. Por lo tanto, la evaluación inicial de la CCB oculta debe realizarse durante la cirugía.

\section{Predictores clínicos de CCB oculta ${ }^{4,7}$ (Tabla 2)}

- Nivel de ALP > 144 U/1.

- Nivel de gamma-glutamiltransferasa (GGT) $>34,5 \mathrm{U} / \mathrm{L}$.

- Nivel total de bilirrubina $>0,8 \mathrm{mg} / \mathrm{dL}$.
- Nivel de bilirrubina directa $>0,4 \mathrm{mg} / \mathrm{dL}$.

- Diámetro del quiste $>8,5 \mathrm{~cm}$.

- Quistes tipo II y IV (WHO-IWGE).

En la Figura 4 se puede ver el algoritmo diagnóstico y terapéutico para la evaluación preoperatoria de la CCB.

\section{Evaluación intraoperatoria}

Una CCB franca suele ser fácil de diagnosticar desde el punto de vista intraoperatorio. Se ha reportado que cuando una comunicación es más ancha que $5 \mathrm{~mm}$, se puede visualizar material hidatídico en el sistema biliar en el 65\% de los $\operatorname{casos}^{13}$ (Figura 5).

Se debe suturar la comunicación siempre que sea posible. Si la comunicación se relaciona con los pedículos biliares primarios o secundarios, el cierre con sutura de la comunicación, podría conducir a una obstrucción biliar en las ramas distales. En tales casos, se deberá considerar realizar cirugía de tipo radical, si esto no es factible, se sugiere procedimientos de drenaje biliar tales como colocación de sonda T o CPRE postoperatoria más esfinterotomía ${ }^{6}$.

A diferencia de la CCB franca, la CCB oculta requiere de una evaluación cuidadosa. En la primera

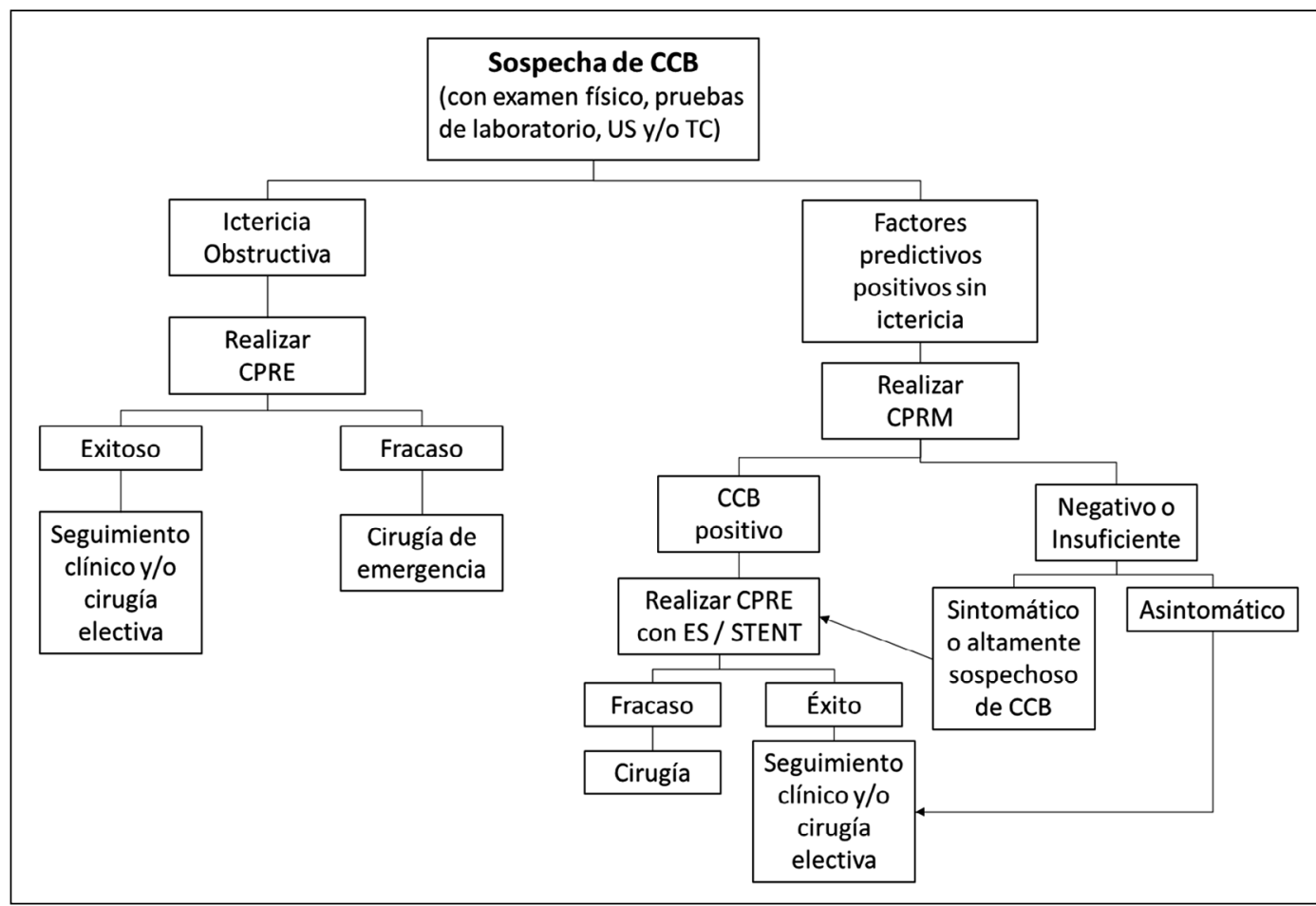

Figura 4. Algoritmo de diagnóstico y terapéutico para la evaluación preoperatoria de CCB. CCB: comunicación cistobiliar. US: ultrasonografía. TC: tomografía computada. CPRE: colangiopancreatografía endoscópica. CPRM: colangiopancreatografía por resonancia magnética. ES: esfinterotomía. 
Figura 5. Colangiohidatidosis. MH: membrana hidatídica; C: colédoco: K1 K2: sonda Kehr; CQ: cavidad quística.

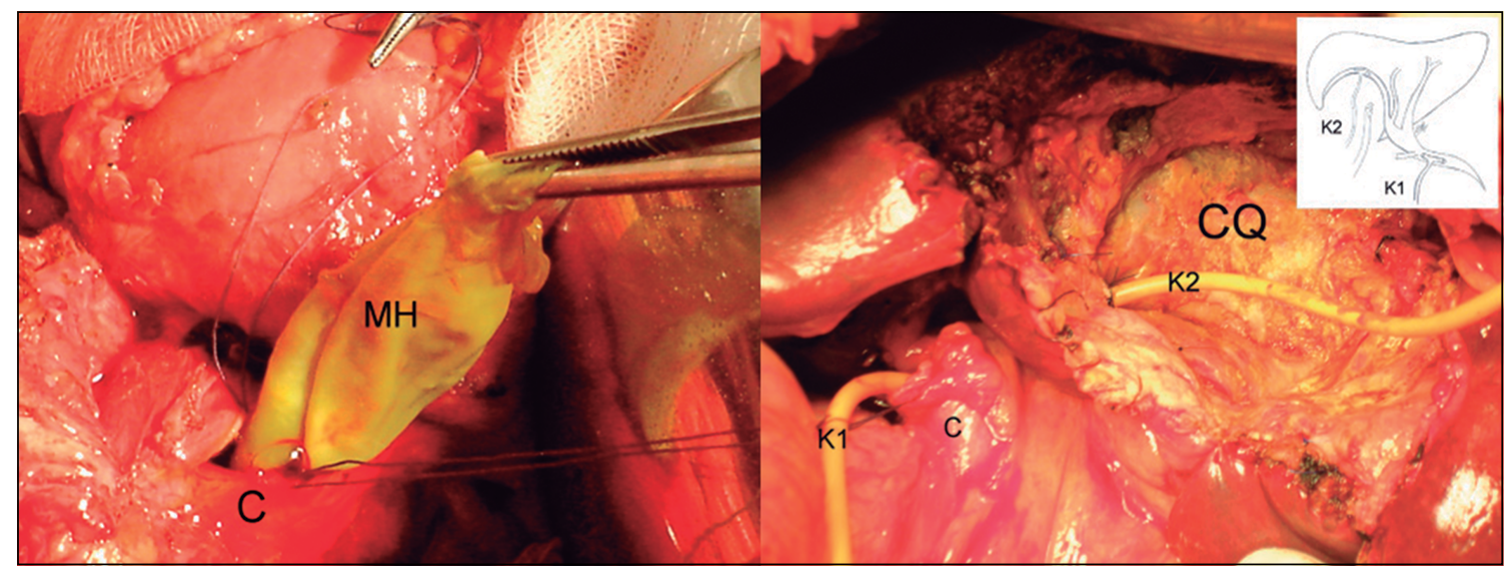

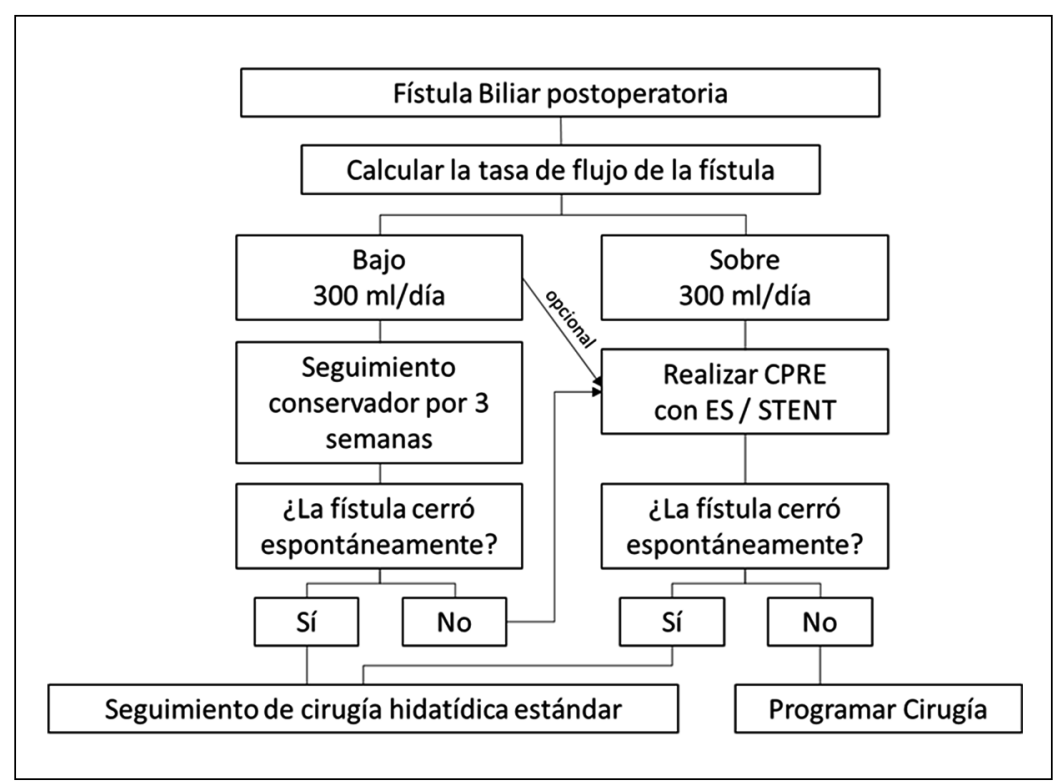

Figura 6. Algoritmo terapéutico de la fuga biliar postoperatoria. CPRE: colangiopancreatografía endoscópica. ES: esfinterotomía.

aspiración del líquido hidatídico, se debe verificar la tinción de bilis. Si el líquido es positivo para bilis, se debe evitar el uso de agentes escolicidas para prevenir la colangitis esclerosante ${ }^{2,3,6}$.

Las lupas quirúrgicas con una ampliación de 2,5 o 3,5 pueden mejorar la calidad de la inspección. En los casos de inspección negativa para detectar fugas biliares, existen varios métodos de evaluación adicional:

- El método más simple es llenar la cavidad con gasa y visualizar si se tiñe.

- Comprimir la vía biliar principal y ver lugar de salida de la bilis.
- Realizar una cisticostomía y a través de ella introducir suero fisiológico y visualizar sitio de salida del mismo.

- Uso de video telescopio para visualizar y suturar comunicaciones inaccesibles ${ }^{14}$.

\section{Evaluación postoperatoria}

Se debe realizar un seguimiento cercano por las eventuales complicaciones que conlleva esta patología:

- En los casos de ictericia postoperatoria, la primera modalidad terapéutica debe ser la CPRE con esfinterotomía (ES) y evacuación de los contenidos hidatídicos dentro de los conductos biliares.

- La complicación postoperatoria más común es la fístula biliar. Cuando esta está presente, es esencial determinar el flujo de la fístula.

- En las fístulas de flujo bajo (< de $300 \mathrm{ml} /$ día $)$ es apropiado el seguimiento conservador durante 3 semanas, ya que la mayoría de estas fístulas cierran espontáneamente en este período. Si la fístula persiste después de 3 semanas, se planifica

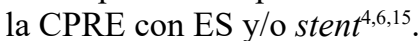

- En las fístulas de alto flujo (> $300 \mathrm{ml} /$ día), el cierre espontáneo es improbable, por lo tanto, la CPRE con ES y/o stent debe realizarse sin demora. La tasa de éxito de la CPRE es muy alta $(90 \%)$, la mayoría de las fístulas cierran en la primera semana ${ }^{4,6,9}$.

- En un pequeño grupo de pacientes en los que fracasan todas las intervenciones, la cirugía radical podría ser una alternativa para la corrección de la fístula biliar. Esto debería ser realizado en un centro con experiencia en cirugía HPB comple$\mathrm{ja}^{3,16}$. 
En nuestra serie de 554 pacientes tratados por hidatidosis hepática, el 26,9\% presentó comunicación a la vía biliar, de los cuales el 16,4\% se presentó como filtración biliar (cierre comunicación biliar antes de los 10 días) y solo el 10,5\% evolucionó como fístula biliar externa ${ }^{17}$.

En la Figura 6 se muestra un algoritmo terapéutico para la evaluación de la fístula biliar postoperatoria.

\section{Conclusión}

La falta del diagnóstico adecuado de ruptura intraquística en la cirugía del quiste hidatídico hepático, ocasiona un aumento de complicaciones postoperatorias. La comunicación cistobiliar (CCB) debe ser sospechada preoperatoriamente y buscada cuidadosamente en el intraoperatorio en presencia de factores predictivos. Todo lo anterior nos llevará al diagnóstico precoz y, por ende, a la elección de una adecuada técnica quirúrgica.

\section{Responsabilidades éticas}

Protección de personas y animales. Los autores declaran que para esta investigación no se han realizado experimentos en seres humanos ni en animales.

Confidencialidad de los datos. Los autores declaran que en este artículo no aparecen datos de pacientes.

Conflictos de interés: no hay.

\section{Bibliografía}

1. Mihmanli M, Idiz UO, Kaya C, Demir U, Bostanci O, Omeroglu S, et al. Current status of diagnosis and treatment of hepatic echinococcosis. World J Hepatol. 2016;8:1169-81.

2. Brunetti E, Kern P, Vuitton DA, Writing Panel for the WHO-IWGE. Expert consensus for the diagnosis and treatment of cystic and alveolar echinococcosis in humans. Acta Trop. 2010;114:1-16.

3. Brunetti E, Kern P, Vuitton DA, Writing Panel for the WHO-IWGE. Expert consensus for the diagnosis and treatment of cystic and alveolar echinococcosis in humans. Acta Trop. 2010;114:1-16.

4. Sozuer E, Akyuz M, Akbulut S. Open surgery for hepatic hydatid disease. Int Surg. 2014;99:764-9.

5. Demircan O, Baymus M, Seydaoglu G, Akinoglu A, Sakman G. Occult cystobiliary communication presenting as postoperative biliary leakage after hydatid liver surgery: Are there significant preoperative clinical predictors? Can J Surg. 2006;49:177-84.

6. Atahan K, Kupeli H, Deniz M, Gur S, Cokmez A, Tarcan E. Can occult cystobiliary fistulas in hepatic hydatid disease be predicted before surgery? Int J Med Sci. 2011;8:

315-20.

7. Ramia JM, Figueras J, De la Plaza

R, Garcia-Parreno J. Cystobiliary communication in liver hydatidosis. Langenbecks Arch Surg. 2012;397: 881-7.

8. Atli M, Kama NA, Yuksek YN, Doganay

M, Gozalan U, Kologlu M, et al. Intrabiliary rupture of a hepatic cyst: Associated clinical factors and proper management. Arch Surg. 2001;136:124955.

9. Zeybek N, Dede H, Balci D, Coskun AK, Ozerhan IH, Peker S, et al. Biliary fistula after treatment for hydatid disease of the liver: When to intervene. World J Gastroenterol. 2013;19:355-61.

10. Dolay K, Akbulut S. Role of endoscopic retrograde cholangiopancreatography in the management of hepatic hydatid disease. World J Gastroenterol. 2014;20:15253-61.

11. Pinto P, Gaete S, Vega P. Utilidad de la CPRE en el diagnóstico y manejo de las complicaciones biliares de la enfermedad hidatídica hepática. Rev Chil Cir. 2016; 68:283-8.

12. Ozaslan E, Bayraktar Y. Endoscopic therapy in the management of hepatobiliary hydatid disease. J Clin Gastroenterol. 2002;35:160-74.
13. Spiliadis C, Georgopoulos S, Dailianas A, Konstantinidis A, Rimikis M, Skandalis $\mathrm{N}$. The use of ERCP in the study of patients with hepatic echinococcosis before and after surgical intervention Gastrointest Endosc. 1996;43:575-9.

14. Zaouche A, Haouet K, Jouini M, El Hachaichi A, Dziri C. Management of liver hydatid cysts with a large biliocystic fistula: Multicenter retrospective study. Tunisian Surgical Association. World J Surg. 2001;25:28-39.

15. Ozmen MM, Coskun F. New technique for finding the ruptured bile duct into the liver cysts: Scope in the cave technique. Surg Laparosc Endosc Percutan Tech. 2002; 12:187-9.

16. El Nakeeb A, Salem A, El Sorogy M, Mahdy Y, Ellatif MA, Moneer A, et al. Cystobiliary communication in hepatic hydatid cyst: Predictors and outcome. Turk J Gastroenterol. 2017;28:125-30.

17. Akcakaya A, Sahin M, Karakelleoglu A, Okan I. Endoscopic stenting for selected cases of biliary fistula after hepatic hydatid surgery. Surg Endosc. 2006;20:1415-8

18. Pinto P, López E. Evolución natural de la fistula biliar externa post cirugía del quiste hidatídico hepático. Rev Chil Cir. 2010;62:476-9. 